\title{
Three-dimensional ghost-fluid large-scale numerical investigation on air explosion
}

\author{
Cheng Wang ${ }^{\mathrm{a}}$, JianXu Ding ${ }^{\mathrm{a}}$, Chi-Wang Shu ${ }^{\mathrm{b}, *}$, Tao $\mathrm{Li}^{\mathrm{a}}$ \\ ${ }^{a}$ State Key Laboratory of Explosion Science and Technology, Beijing Institute of Technology, Beijing \\ 100081, China \\ ${ }^{b}$ Division of Applied Mathematics, Brown University, Providence, RI 02912, USA
}

\begin{abstract}
Based on the double shockwave approximation procedure, a local Riemann solver for strongly nonlinear equations of state (EOS) such as the Jones-Wilkins-Lee (JWL) EOS is presented, which has suppressed successfully numerical oscillation caused by high-density ratio and high-pressure ratio across the interface between explosion products and air. The real ghost fluid method (RGFM) and the level set method have been used for converting multimedium flows into pure flows and for implicitly tracking the interface, respectively. A fifth order finite difference weighted essentially non-oscillatory (WENO) scheme and a third order TVD Runge-Kutta method are utilized for the spatial discretization and the time advance, respectively. An enclosed-type MPI-based parallel methodology for the RGFM procedure on a uniform structured mesh is presented to realize the parallelization of three-dimensional (3D) air explosion. The overall process of 3D air explosion of both TNT and aluminized explosives has been successfully simulated. The overpressures at different locations of 3D air explosion for both explosives mentioned above are monitored and analyzed for revealing the influence of aluminum powder combustion on the overpressure of the explosion wave. Numerical results indicate that, due to aluminum powder afterburning, the attenuation of the explosion wave formed by aluminized explosives is slower than that caused by TNT. Keywords: Air explosion, WENO scheme, RGFM, local Riemann solver, parallel computation
\end{abstract}

*Corresponding author: shu@dam.brown.edu

(C) 2016. This manuscript version is made available under the Elsevier user license

http://www.elsevier.com/open-access/userlicense/1.0/

Preprint submitted to Elsevier

May 27, 2016 


\section{Introduction}

Air explosion is a typical multi-medium problem, in which the explosion flow field usually consists of many media such as detonation products and air. It is obvious that the sharp medium interface with high-density ratio and high-pressure ratio separates detonation products from air. In the numerical simulation of air explosion, because of the abrupt change of density and pressure close to the interface, unphysical numerical oscillation may easily appear in the neighborhood of the interface. Meanwhile, with the continuous upgrade of explosives, density ratio and pressure ratio increase constantly and significantly. Therefore, tracking and treating the strong nonlinear discontinuous interface in air explosion have received considerable attention.

Many interface tracking techniques have been discussed over the past few decades. The marker-and-cell (MAC) method proposed by Harlow and Welch [1] has often been used to track interface movement and flow field evolution. However, the maintenance of a sharp interface among multi-medium is difficult. Hirt and Nichols [2] presented another interface tracking algorithm known as volume-of-fluid (VOF) method without tracking the motion of the multi-medium interface, in which, as time increases, the volume of each medium in each cell is changing continuously and the new interface is reconstructed from the volume at the current time level. The main drawback of the VOF method is that, for deciding the normal direction of the multi-medium interface, a cluster of eight neighboring cells for the two dimensional case must be taken into consideration [3]. To overcome such drawbacks of the VOF method, the moment of fluid (MOF) method introduced by Ahn and Shashkov [4] and by Dyadechko and Shashkov [5] with second order accuracy employed not only volume fractions but also the position of the centroid, which allows the MOF method to utilize the cell where reconstruction is performed. The well-known particle-in-cell (PIC) algorithm by Amsden [6] is a Lagrangian description of the flows in which particles are explicitly associated with different medium and thus the interface can be tracked easily. The principal drawbacks are a large numerical diffusion and a numerical noise, due to the momentum transfer between grids and particles and the employment of finite particle numbers. The 
level set method developed by Osher and Sethian [7] is a simple and versatile method for tracking the evolution of a locomotive interface. We would like to mention in particular the work of Vahab and Miller [8], which provided a second order front-tracking method by combining the Godunov algorithm with the level-set method, in which global conservation was realized. The level-set method $[9,10,11]$ generally uses a Hamilton-Jacobi equation to describe the moving interface, which is tracked as the zero level set of a continuous function mimicking the signed distance function instead of an explicit function at the new time level. Thus, cases with complex interfaces, such as crisscross, torsion and separation, etc., can be easily handled by the level-set method.

Fedkiw et al. [12] presented the original ghost fluid method (OGFM), which can address excellently the interaction between a weak shock wave and an interface. To further improve the accuracy of the OGFM method, Liu et al. [13] discussed the modified ghost fluid method (MGFM) procedure, which performed better than OGFM due to a local Riemann solver, where the status of the ghost fluid across the interface for each phase was defined by predicted interfacial status. The accuracy of MGFM for gas-gas Riemann problem performed by Liu et al. in [14] shows that, compared with the exact solution of a Riemann problem, the MGFM solution could reach second order accuracy near multi-medium interface. The key point of the real ghost fluid method (RGFM) procedure, presented by Wang et al. [15] was that, according to the status of the medium across the interface, a local Riemann problem was constructed at first and interfacial status obtained was then used to redefine flow status for not only the real fluid grid points close to the interface but also the ghost fluid grid points, by which smaller error was introduced in the RGFM procedure in comparison to the MGFM procedure. We remark that the RGFM method we adopt in this paper does have conservation errors, like other ghost fluid type methods, but it seems to have smaller conservation errors than OGFM or MGFM in numerical simulation. We refer the readers to [15] for a more detailed discussion on this issue.

Since OGFM, MGFM and RGFM were introduced, an increasing number of numerical simulations combining these methods for multi-medium flows can be found in the literature. However, most of the previous simulations with GFM-based procedures used simple and 
linear equation of state (EOS) for describing multi-medium flows such as explosion products and air in air explosion, while the complex EOS such as the Jones-Wilkins-Lee (JWL) EOS have seldomly been used. Meanwhile, numerical simulations based on the RGFM procedure splitting a multi-medium problem into pure flows usually require large parallel computer resources. In general, serial computation cannot meet the requirements of multi-dimensional large-scale engineering applications.

In this paper, based on a double shockwave approximation procedure, we first present in detail a technique to construct and solve the local Riemann problem with the complex EOS such as JWL used in air explosion. Our numerical simulation demonstrates that this technique effectively eliminates unphysical oscillation which often occurs at the multi-medium interface in explosion flow fields. Combining the RGFM method, formally transforming multi-medium flow into pure flows with an enclosed-type parallelization module, the mechanisms of 3D air explosion are studied by using a fifth order finite difference WENO scheme on a uniform structured mesh.

\section{Governing equations}

The governing equations in a conservative form without considering the viscous and thermal diffusion effects for describing a 3D air explosion can be written as

$$
\begin{aligned}
& \frac{\partial U}{\partial t}+\frac{\partial F(U)}{\partial x}+\frac{\partial G(U)}{\partial y}+\frac{\partial H(U)}{\partial z}=0 \\
& U=(\rho, \rho u, \rho v, \rho w, \rho E)^{T} \\
& F(U)=\left(\rho u, \rho u^{2}+p, \rho u v, \rho u w,(\rho E+p) u\right)^{T} \\
& G(U)=\left(\rho v, \rho u v, \rho v^{2}+p, \rho v w,(\rho E+p) v\right)^{T} \\
& H(U)=\left(\rho w, \rho u w, \rho v w, \rho w^{2}+p,(\rho E+p) w\right)^{T}
\end{aligned}
$$

where $\rho$ and $p$ denote the density and pressure, respectively. $u, v$ and $w$ are velocity components in the $x, y$ and $z$ directions in Eulerian coordinates, and the total energy $E$, generally consisting of internal energy and kinetic energy, is given as

$$
E=\frac{u^{2}+v^{2}+w^{2}}{2}+e
$$

where $e$ is the internal energy per unit mass. 
Tab. 1: JWL EOS parameters for explosion products of TNT charge.

\begin{tabular}{llllll}
\hline$\rho_{0}\left(\mathrm{~kg} / \mathrm{m}^{3}\right)$ & $A(\mathrm{MPa})$ & $B(\mathrm{MPa})$ & $R_{1}$ & $R_{2}$ & $\omega$ \\
\hline 1630.0 & 373800.0 & 3747.0 & 4.15 & 0.9 & 0.35 \\
\hline
\end{tabular}

For aluminized explosives, the Miller model [16] describing the combustion and heat release process of aluminum powder can be used to describe the reaction process. By coupling the above 3D Euler equations with the Miller model, the whole process of air explosion for aluminized explosives can be captured numerically. The Miller model given in [16] is

$$
\frac{d \lambda}{d t}=\frac{1}{4}(1-\lambda)^{\frac{1}{2}} p^{\frac{1}{6}}
$$

where $\frac{d \lambda}{d t}$ is the material derivative and $\lambda$ is the chemical reaction process variable characterizing the aluminum powder reaction degree. The reaction process parameters $\lambda=0$ and $\lambda=1$ are defined as the initially unreacted and completely burned states, respectively. The material derivative in (3) is first converted to Eulerian derivatives and then (3) is solved together with the governing equations (1) using the WENO scheme described below. To close the above governing equations, the respective EOS for air and explosion products should be introduced. The ideal gaseous EOS for air can be presented as

$$
p=(\gamma-1) \rho e
$$

where $\gamma=1.4$ is a gaseous constant.

Assuming no heat loss to the surroundings, the expansion of explosion products of TNT charge is usually described by the JWL EOS [17], which can be expressed in the following form

$$
p=A\left(1-\frac{\omega \rho}{R_{1} \rho_{0}}\right) e^{-\frac{R_{1} \rho_{0}}{\rho}}+B\left(1-\frac{\omega \rho}{R_{2} \rho_{0}}\right) e^{-\frac{R_{2} \rho_{0}}{\rho}}+\omega \rho e
$$

where the parameters $A, B, R_{1}, R_{2}, \omega$ and $\rho_{0}$ are material constants of detonation products, which are shown in Table 1.

The EOS of detonation products of aluminized explosives should be able to establish the essential relationship among pressure, density, internal energy and the reaction process vari- 
Tab. 2: JWL-Miller EOS parameters for explosion products of aluminized explosives.

\begin{tabular}{lllllll}
\hline$A(\mathrm{MPa})$ & $B(\mathrm{MPa})$ & $R_{1}$ & $R_{2}$ & $\omega$ & $\rho_{0}\left(\mathrm{~kg} / \mathrm{m}^{3}\right)$ & $Q(\mathrm{MPa})$ \\
\hline 695130.0 & 3130.0 & 5.4 & 1.4 & 0.4 & 1880 & 11000 \\
\hline
\end{tabular}

able. The JWL-Miller EOS [16] for describing detonation products of aluminized explosives are adopted as

$$
p=A\left(1-\frac{\omega \rho}{R_{1} \rho_{0}}\right) e^{-\frac{R_{1} \rho_{0}}{\rho}}+B\left(1-\frac{\omega \rho}{R_{2} \rho_{0}}\right) e^{-\frac{R_{2} \rho_{0}}{\rho}}+\frac{\omega \rho(\widetilde{E}+\lambda Q)}{\rho_{0}}
$$

where $\widetilde{E}$ denotes the energy content of aluminized explosives for keeping the so-called CJ condition, which denotes a relationship between the propagating velocity of the detonation wave and the local speed of sound. The constant $Q$ is defined as afterburning energy release after the CJ plane located at the end of the reaction zone. $A, B, R_{1}, R_{2}, \omega$ and $\rho_{0}$ in the above equation (6) are material constants of detonation products, which can be found in Table 2.

The semi-discrete approximation of equation (1) is given by

$$
\begin{aligned}
\left(\frac{\partial U}{\partial t}\right)_{i, j, k}= & -\frac{1}{\Delta x}\left(\hat{F}_{i+\frac{1}{2}, j, k}-\hat{F}_{i-\frac{1}{2}, j, k}\right)-\frac{1}{\Delta y}\left(\hat{G}_{i, j+\frac{1}{2}, k}-\hat{G}_{i, j-\frac{1}{2}, k}\right) \\
& -\frac{1}{\Delta z}\left(\hat{H}_{i, j, k+\frac{1}{2}}-\hat{H}_{i, j, k-\frac{1}{2}}\right)
\end{aligned}
$$

where $\hat{F}_{i+\frac{1}{2}, j, k}, \hat{G}_{i, j+\frac{1}{2}, k}$ and $\hat{H}_{i, j, k+\frac{1}{2}}$ denote numerical fluxes, which are obtained by a fifth order finite difference WENO scheme with the Lax-Friedrichs splitting [18]. $\Delta x, \Delta y$ and $\Delta z$ are defined as spatial step sizes in the $\mathrm{x}, \mathrm{y}$ and $\mathrm{z}$ directions respectively. A third order TVD Runge-Kutta scheme [19] is used to integrate the system of ordinary differential equations (7) in time, which is described as

$$
\begin{aligned}
U^{(1)} & =U^{n}+\Delta t^{n} L\left(U^{n}, t^{n}\right) \\
U^{(2)} & =\frac{3}{4} U^{n}+\frac{1}{4} U^{(1)}+\frac{1}{4} \Delta t^{n} L\left(U^{(1)}, t^{n}+\Delta t^{n}\right) \\
U^{n+1} & =\frac{1}{3} U^{n}+\frac{2}{3} U^{(2)}+\frac{2}{3} \Delta t^{n} L\left(U^{(2)}, t^{n}+\frac{1}{2} \Delta t^{n}\right)
\end{aligned}
$$

where $L(\cdot, \cdot)$ denotes the operator defined by the right hand side of $(7)$. A parallel adaptive code to solve $3 \mathrm{D}$ detonation problems is developed in [20]. 


\section{Riemann solver for air explosion}

The level set technique used in this paper provides an efficient method to track multimedium interface. The level set function $\phi$ at any node attempts to describe the signed normal distance from the interface with $\phi<0$ inside the medium of interest and $\phi>0$ outside. The level set $\phi=0$ always implicitly represents the medium interface. The level set advection equation propagating the interface can be written as

$$
\frac{\partial \phi}{\partial t}+u \frac{\partial \phi}{\partial x}+v \frac{\partial \phi}{\partial y}+w \frac{\partial \phi}{\partial z}=0
$$

A fifth order WENO scheme [18, 21, 22] combined with a third order TVD Runge-Kutta [19] is employed to solve equation (9). The reinitialization is a necessary step in the level set method to keep the level set function close to the signed distance function [23, 24, 25, 26].

The implicit level-set function can track the specific location of the interface between explosion products and air. Taking a one-dimensional problem as an example, we first assume the interface exists between nodes $i$ and $i+1$ as shown in Fig. 1. A very simple and efficient definition for Riemann initial constant status is $U_{L}=U_{i}$ and $U_{R}=U_{i+1}$, which can be conveniently implemented in 3D air explosion. Thus interfacial status including pressure $p_{*}$, velocity $u_{*}$ and $\rho_{* L}$ and $\rho_{* R}$ meaning left and right sides of densities across the interface can be derived by the two-shock approximation procedure discussed next. As for the fifth order WENO finite difference scheme, three ghost nodes in each direction should be defined artificially. For the left medium in Fig. 1, the key technique of the RGFM method is to assign the interfacial status $p_{*}, u_{*}$ and $\rho_{* L}$ to the nodes from $i$ to $i+3$. A similar operation can be applied for the right medium in addition to assign $\rho_{* R}$ from node $i-2$ to node $i+1$. Thus the multi-medium flow is changed into two pure flows, which can be computed respectively using the traditional high order WENO scheme. Next we would like to describe briefly the extension of the one-dimensional Riemann construction to 3D. Considering a node inside a band of $|\phi|<1.5 \times \min (\Delta x, \Delta y, \Delta z)$ in the explosion products, another node contained in the same band but on the opposite side of the interface must be chosen to make the angle

formed by the respective normals at both nodes minimum. Thus the Riemann problem in the normal direction using the above nodes can be constructed. Once the intermediate status 
are obtained by the following double-shock approximation, the flow status at those real fluid nodes neighboring the interface are redefined. The ghost fluid status are then obtained by solving an extension equation using the WENO scheme and TVD Runge-Kutta method, the details of which can be found in [11].

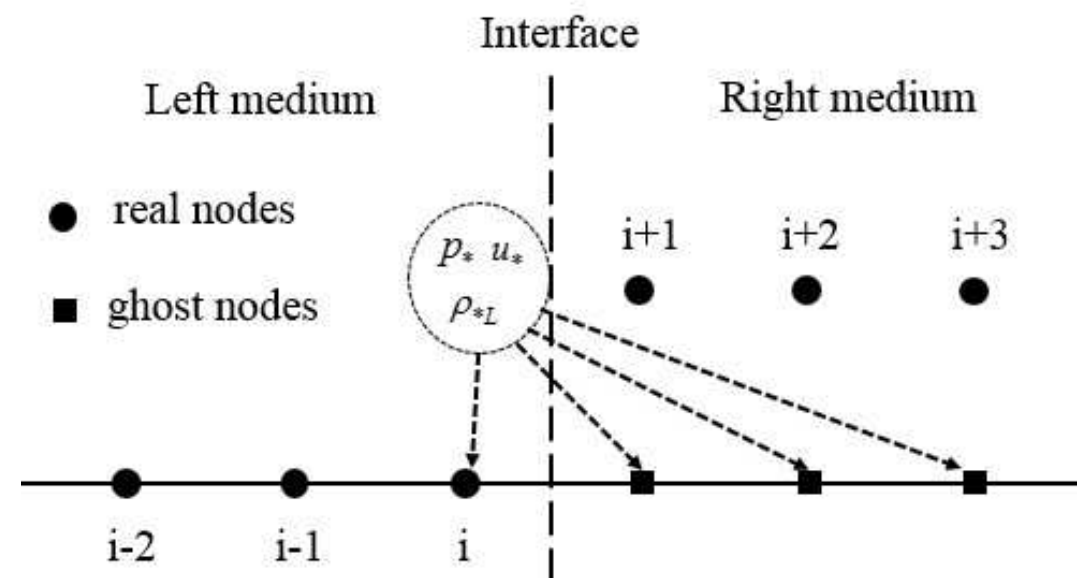

Fig. 1: Redefining the real and ghost nodes for the left medium.

Next, the solution to a local Riemann problem in the air explosion will be presented in detail. Taking the one dimensional case as an example, we define the pre-shock values $\rho_{k}$, $u_{k}$ and $p_{k}$ as the Riemann initial constant status. The post-shock values are $\rho_{* k}, u_{*}$ and $p_{*}$. Here the notation $k$ stands for $L$ or $R$ appearing in the following formulas. Note that $\rho_{* L}$ and $\rho_{* R}$ are densities on both sides of the interface.

The Rankine-Hugoniot conditions can be given as

$$
\begin{aligned}
& \rho_{k}\left(u_{k}-S_{k}\right)=\rho_{* k}\left(u_{*}-S_{k}\right) \\
& \rho_{k}\left(u_{k}-S_{k}\right)^{2}+p_{k}=\rho_{* k}\left(u_{*}-S_{k}\right)+p_{*} \\
& \rho_{k}\left(u_{k}-S_{k}\right)\left(\frac{1}{2}\left(u_{k}-S_{k}\right)^{2}+e_{k}+\frac{p_{k}}{\rho_{k}}\right)=\rho_{*}\left(u_{*}-S_{k}\right)\left(\frac{1}{2}\left(u_{*}-S_{k}\right)^{2}+e_{*}+\frac{p_{*}}{\rho_{*}}\right)
\end{aligned}
$$

where $S_{k}$ denotes the speed of the left- or right-facing shock wave.

With the help of the first and second equations in (10), the relationship bridging velocity and pressure of the interface can be obtained as

$$
\begin{aligned}
& u_{*}=F_{1}\left(p_{*}\right)=u_{L}-\sqrt{\left(p_{*}-p_{L}\right)\left(\frac{1}{\rho_{L}}-\frac{1}{\rho_{* L}}\right)} \\
& u_{*}=F_{2}\left(p_{*}\right)=u_{R}+\sqrt{\left(p_{*}-p_{R}\right)\left(\frac{1}{\rho_{R}}-\frac{1}{\rho_{* R}}\right)}
\end{aligned}
$$


here $\rho_{* L}$ and $\rho_{* R}$ are still unknowns for the time being and need to be solved.

A nonlinear function relationship with respect to the interface pressure is obtained by the two equations above, which can be easily written as

$$
F\left(p_{*}\right)=F_{1}\left(p_{*}\right)-F_{2}\left(p_{*}\right)=0
$$

Combining the energy conservation equation with the EOS, an implicit function with regard to $\rho_{* L}, \rho_{* R}$ and $p_{*}$ can be deduced as

$$
\begin{aligned}
& e_{L}\left(p_{L}, \rho_{L}\right)-e_{* L}\left(p_{*}, \rho_{* L}\right)-\frac{1}{2}\left(p_{L}+p_{*}\right) \frac{\rho_{L}-\rho_{* L}}{\rho_{L} \rho_{* L}}=0 \\
& e_{* R}\left(p_{*}, \rho_{* R}\right)-e_{R}\left(p_{R}, \rho_{R}\right)-\frac{1}{2}\left(p_{*}+p_{R}\right) \frac{\rho_{* R}-\rho_{R}}{\rho_{* R} \rho_{R}}=0
\end{aligned}
$$

For the local Riemann problem comprising of the JWL and the ideal gaseous EOS, the process of solving equation (13) by a Newton iterative method is described below. It is first supposed that, for the one dimensional Riemann problem, explosion products are located at the left side of the interface and air is on the right side. Obviously, (13), (14) and (15) are now a system of nonlinear equations consisting of the interface pressure and densities on both sides of interface as unknown variables. Thus the classical Newton iterative method could be used as follows

$$
p_{*(n+1)}=p_{*(n)}-\frac{F_{1}\left(p_{*(n)}\right)-F_{2}\left(p_{*(n)}\right)}{F_{1}^{\prime}\left(p_{*(n)}\right)-F_{2}^{\prime}\left(p_{*(n)}\right)}
$$

The initial guess value of $p_{*}$ is necessary for the Newton iteration, and should be selected properly. When the given value is close enough to the true solution, the rate of convergence is second order. In our simulations, the arithmetic average between the left and right pressures is set to the initial guess value for the iteration. The JWL EOS is plugged into equation (14), and then the following implicit relationship can be given

$$
\begin{aligned}
& \left(p_{L}-A\left(1-\frac{\omega \rho_{L}}{R_{1} \rho_{0}}\right) e^{-\frac{R_{1} \rho_{0}}{\rho_{L}}}-B\left(1-\frac{\omega \rho_{L}}{R_{2} \rho_{0}}\right) e^{-\frac{R_{2} \rho_{0}}{\rho_{L}}}\right) \rho_{* L} \\
& -\left(p_{*}-A\left(1-\frac{\omega \rho_{* L}}{R_{1} \rho_{0}}\right) e^{-\frac{R_{1} \rho_{0}}{\rho_{* L}}}-B\left(1-\frac{\omega \rho_{* L}}{R_{2} \rho_{0}}\right) e^{-\frac{R_{2} \rho_{0}}{\rho_{* L}}}\right) \rho_{L}-\frac{1}{2} \omega\left(p_{L}+p_{*}\right)\left(\rho_{L}-\rho_{* L}\right)=0
\end{aligned}
$$

With an appropriate initial guess $p_{*}$, equation (17) becomes a nonlinear equation for the dependent variable $\rho_{* L}$, which also needs to be solved using the Newton iterative method. 
In the process of iteration, the derivative of equation (17) with respect to density can be written as

$$
\begin{aligned}
\frac{\partial f}{\partial \rho_{* L}}= & p_{L}+\rho_{L}\left(\frac{A e^{-\frac{R_{1} \rho_{0}}{\rho_{* L}}}\left(R_{1} \rho_{0}-\omega \rho_{* L}\right)}{\rho_{* L}^{2}}+\frac{B e^{-\frac{R_{2} \rho_{0}}{\rho_{* L}}}\left(R_{2} \rho_{0}-\omega \rho_{* L}\right)}{\rho_{* L}^{2}}\right) \\
& -\rho_{L}\left(\frac{A \omega e^{-\frac{R_{1} \rho_{0}}{\rho_{* L}}}}{R_{1} \rho_{0}}+\frac{B \omega e^{-\frac{R_{2} \rho_{0}}{\rho_{* L}}}}{R_{2} \rho_{0}}\right)+\omega\left(\frac{p_{L}}{2}+\frac{p_{*}}{2}\right) \\
& -\frac{A e^{-\frac{R_{1} \rho_{0}}{\rho_{L}}}\left(R_{1} \rho_{0}-\omega \rho_{L}\right)}{R_{1} \rho_{0}}-\frac{B e^{-\frac{R_{2} \rho_{0}}{\rho_{L}}}\left(R_{2} \rho_{0}-\omega \rho_{L}\right)}{R_{2} \rho_{0}}
\end{aligned}
$$

Similarly, for the ideal gaseous EOS, we have

$$
\frac{p_{*}}{\gamma-1}-\frac{p_{R} \rho_{* R}}{(\gamma-1) \rho_{R}}-\frac{1}{2}\left(p_{*}+p_{R}\right) \frac{\rho_{* R}-\rho_{R}}{\rho_{R}}=0
$$

The derivative of equation (19) with respect to density can be given easily as

$$
\frac{\partial f}{\partial \rho_{* R}}=-\frac{p_{R}+p_{*}}{2 \rho_{R}}-\frac{p_{R}}{\rho_{R}(\gamma-1)}
$$

Now we substitute both pressure $p_{*}$ and density $\rho_{* L}$ obtained by the iterative method into equation (11) to produce the expression $F_{1}\left(p_{*}\right)$. In the same way, substitution of the given $p_{*}$ and density $\rho_{* R}$ into equation (12) yields $F_{2}\left(p_{*}\right)$. Apparently, derivatives of functions $F_{1}\left(p_{*}\right)$ and $F_{2}\left(p_{*}\right)$ with respect to the interface pressure $p_{*}$ are

$$
\begin{aligned}
& \frac{d F_{1}\left(p_{*}\right)}{d p_{*}}=-\sqrt{-\frac{\frac{1}{\rho_{* L}}-\frac{1}{\rho_{L}}}{p_{*}-p_{L}}}-\frac{\frac{\frac{\rho_{* L}^{\prime}\left(p_{*}-p_{L}\right)}{\rho_{* L}^{2}}+\frac{1}{\rho_{* L}}-\frac{1}{\rho_{L}}}{p_{*}-p_{L}}}{2 \sqrt{-\frac{\frac{1}{\rho_{*}}-\frac{1}{\rho_{L}}}{p_{*}-p_{L}}}} \\
& \frac{d F_{2}\left(p_{*}\right)}{d p_{*}}=\sqrt{-\frac{\frac{1}{\rho_{* R}}-\frac{1}{\rho_{R}}}{p_{*}-p_{R}}}+\frac{\frac{\frac{\rho_{* R}^{\prime}\left(p_{*}-p_{R}\right)}{\rho_{* R}^{2}}+\frac{1}{\rho_{* R}}-\frac{1}{\rho_{R}}}{p_{*}-p_{R}}}{2 \sqrt{-\frac{1}{\rho_{* R}-\frac{1}{\rho_{R}}}}}
\end{aligned}
$$

By a direct calculation of the derivatives of densities $\rho_{* L}$ and $\rho_{* R}$ with respect to the interface pressure, we arrive at the desired results as follows

$$
\begin{aligned}
\frac{d \rho_{* L}}{d p_{*}}= & \frac{\rho_{L}+\frac{\omega\left(\rho_{L}-\rho_{* L}\right)}{2}}{p_{L}+\rho_{L}\left(\frac{A e^{-\frac{R_{1} \rho_{0}}{\rho_{* L}}}\left(R_{1} \rho_{0}-\omega \rho_{* L}\right)}{\rho_{* L}^{2}}+\frac{B e^{-\frac{R_{2} \rho_{0}}{\rho_{* L}}}\left(R_{2} \rho_{0}-\omega \rho_{* L}\right)}{\rho_{* L}^{2}}-\frac{A \omega e^{-\frac{R_{1} \rho_{0}}{\rho_{* L}}}}{R_{1} \rho_{0}}-\frac{B \omega e^{-\frac{R_{2} \rho_{0}}{\rho_{* L}}}}{R_{2} \rho_{0}}\right)} \\
& +\omega\left(\frac{p_{L}}{2}+\frac{p_{*}}{2}\right)-\frac{A e^{-\frac{R_{1} \rho_{0}}{\rho_{L}}}\left(R_{1} \rho_{0}-\omega \rho_{L}\right)}{R_{1} \rho_{0}}-\frac{B e^{-\frac{R_{2} \rho_{0}}{\rho_{L}}}\left(R_{2} \rho_{0}-\omega \rho_{L}\right)}{R_{2} \rho_{0}}
\end{aligned}
$$




$$
\frac{d \rho_{* R}}{d p_{*}}=-\frac{\frac{1}{\gamma-1}-\frac{1}{2}\left(\frac{\rho_{* R}}{\rho_{R}}-1\right)}{\frac{p_{R}+p_{*}}{2 \rho_{R}}+\frac{p_{R}}{\rho_{R}(\gamma-1)}}
$$

By substituting $F_{1}\left(p_{*}\right), F_{2}\left(p_{*}\right), F_{1}^{\prime}\left(p_{*}\right)$ and $F_{2}^{\prime}\left(p_{*}\right)$ obtained above into equation $(16)$, the pressure $p_{*(n+1)}$ is obtained, which will be used as a starting value for the next iteration. If $\left|p_{*(n+1)}-p_{*(n)}\right|$ is smaller than a threshold, Newton iteration will be stopped, and the final

result $p_{*(n+1)}$ is recorded as pressure $p_{*}$. Then, the velocity and the two densities can be obtained by simultaneously solving (11), (14) and (15). Thus, solution to the local Riemann problem in air explosion is completed. The interfacial normal velocities of all nodes bordering the interface are given by the Riemann solver. The extension equation [27] can be used to obtain the normal velocity of the ghost nodes. We advance the level set equation to the next time step utilizing the new velocity field.

\section{Parallelization for the RGFM procedure}

For parallel implementation, the computational domain should first be divided into many small subdomains. That is to say, each processor is only responsible for computing the corresponding subdomain, as shown in Fig. 2.

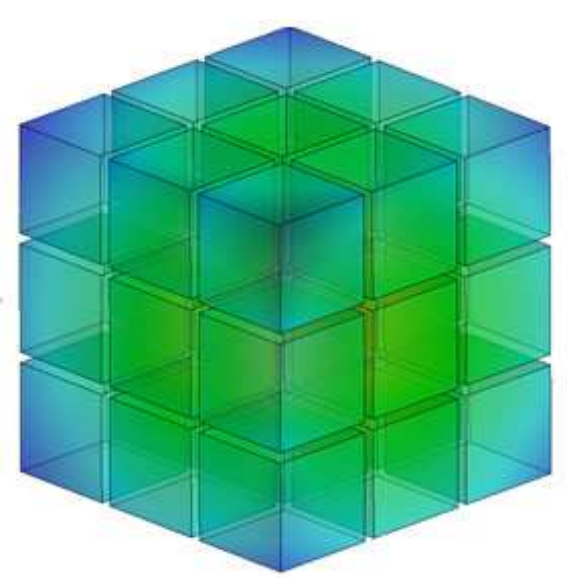

Fig. 2: Computational domain divided equally into subdomains for parallel implementation.

When calculating the spatial derivatives at any grid point, it is necessary to obtain the values of its adjacent nodes for interpolation. The template for the fifth order WENO scheme requires seven grid points, which is obviously wider than traditional lower order schemes. For such grid points close to the border of any processor, the corresponding information 
required for interpolation may be located in other adjacent processors, as shown in Fig. 3. It can be seen from Fig. 3 that the computation of several columns of grid points located at the right side of the yellow middle processor would need to use information in the right adjacent processor. Therefore, information of the adjacent processors must be transmitted into the current processor for parallel computation.

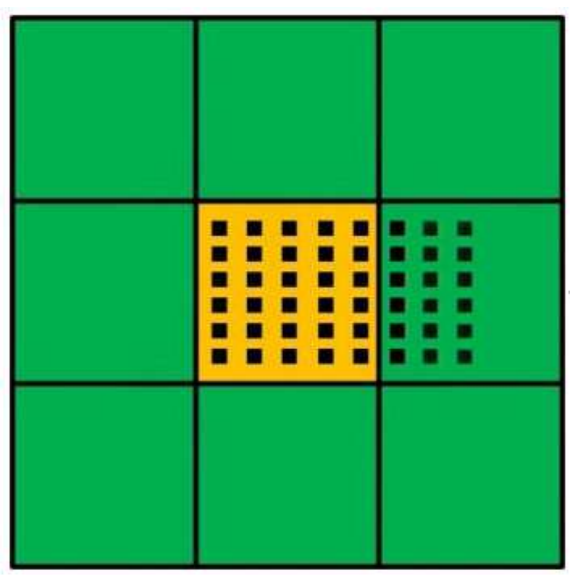

Fig. 3: Data communication required by a finite difference WENO scheme.

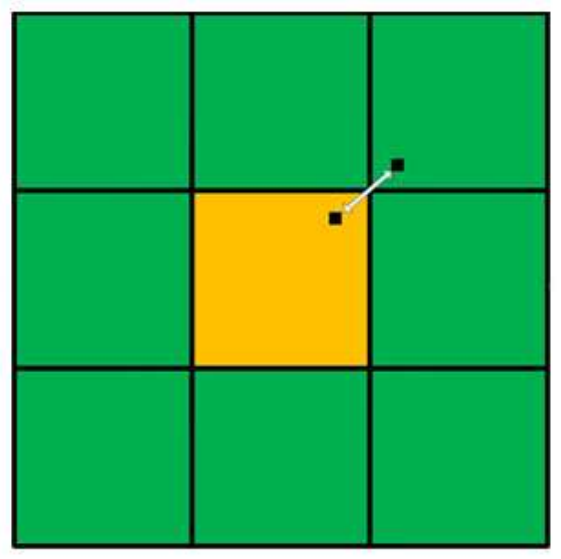

Fig. 4: Possible data communication required by RGFM.

For an ordinary finite difference scheme, it generally only requires data communication of the current processor with its adjacent processors in orthogonal directions. In other words, information in the processors in the diagonal directions is not needed. For the numerical simulations of air explosion based on both RGFM and WENO, however, it may be different. If the interface is very close to a corner of any subdomain, information such as pressure and density at some grid points of an adjacent diagonal processor may be needed in the construc- 
tion of a local Riemann solver, as shown in Fig. 4. Considering such special requirement of data communication for the RGFM method, an enclosed-type data communication mode is presented, in which an enclosed communication layer with a width of three grid points is created on the periphery of any processor. In a 3D flow field, for a processor not located at the border of the computational domain, there are 26 adjacent processors. Therefore, 26 "send" buffers corresponding to 26 "receive" buffers are established. Fig. 5 shows an individual processor. A cubic domain surrounded by the bright cyan lines is computed by the WENO scheme. Others are not only ghost layers but also 26 "receive" buffers. It should be noted that, without loss of generality, the corresponding 26 "send" buffers are not illustrated in Fig. 5.

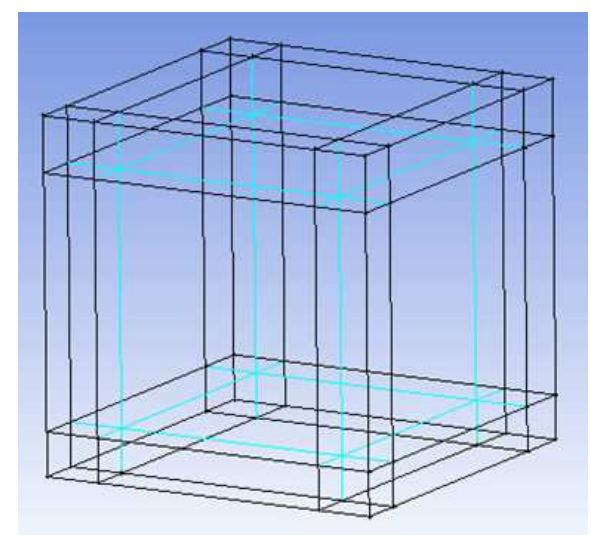

Fig. 5: An enclosed-type data communication style for an individual processor.

Table 3 shows the identifiers of all "send" buffers and their processors corresponding to all "receive" buffers for the $n$-th subdomain, and identifiers of all "receive" buffers and their processors corresponding to all the "send" buffers for the $n$-th subdomain. It is noted that the notations $x$ and $y$ refer to processor numbers in the horizontal and vertical directions respectively.

The classical fifth order WENO scheme used in this paper needs three layers of ghost points for the current processor. As for the RGFM method, three layers of ghost points are also enough when constructing a local Riemann problem. Therefore, an enclosed-type MPI-based parallel procedure containing three layers of ghost nodes is employed in all the following numerical examples. 
Tab. 3: Communication mode for the $n$-th receive (send) processor.

\begin{tabular}{|c|c|c|c|}
\hline $\begin{array}{l}\text { Processor number } \\
\text { of receive (send) buffer }\end{array}$ & $\begin{array}{l}\text { Receive (send) } \\
\text { buffer number }\end{array}$ & $\begin{array}{l}\text { Send (receive) } \\
\text { buffer number }\end{array}$ & $\begin{array}{c}\text { Processor number } \\
\text { of send (receive) buffer }\end{array}$ \\
\hline $\mathrm{n}$ & 1 & 8 & $n-x y-x-1$ \\
\hline $\mathrm{n}$ & 2 & 7 & $n-x y-x+1$ \\
\hline $\mathrm{n}$ & 3 & 6 & $n-x y+x-1$ \\
\hline $\mathrm{n}$ & 4 & 5 & $n-x y+x+1$ \\
\hline $\mathrm{n}$ & 5 & 4 & $n+x y-x-1$ \\
\hline $\mathrm{n}$ & 6 & 3 & $n+x y-x+1$ \\
\hline $\mathrm{n}$ & 7 & 2 & $n+x y+x-1$ \\
\hline $\mathrm{n}$ & 8 & 1 & $n+x y+x+1$ \\
\hline $\mathrm{n}$ & 9 & 12 & n-xy-x \\
\hline $\mathrm{n}$ & 10 & 11 & $n-x y+x$ \\
\hline $\mathrm{n}$ & 11 & 10 & $n+x y-x$ \\
\hline $\mathrm{n}$ & 12 & 9 & $n+x y+x$ \\
\hline $\mathrm{n}$ & 13 & 16 & n-xy-1 \\
\hline $\mathrm{n}$ & 14 & 15 & $n-x y+1$ \\
\hline $\mathrm{n}$ & 15 & 14 & $n+x y-1$ \\
\hline $\mathrm{n}$ & 16 & 13 & $\mathrm{n}+\mathrm{xy}+1$ \\
\hline $\mathrm{n}$ & 17 & 20 & $n-x-1$ \\
\hline $\mathrm{n}$ & 18 & 19 & $n-x+1$ \\
\hline $\mathrm{n}$ & 19 & 18 & $n+x-1$ \\
\hline $\mathrm{n}$ & 20 & 17 & $n+x+1$ \\
\hline $\mathrm{n}$ & 21 & 22 & $n-1$ \\
\hline $\mathrm{n}$ & 22 & 21 & $\mathrm{n}+1$ \\
\hline $\mathrm{n}$ & 23 & 24 & $n-x$ \\
\hline $\mathrm{n}$ & 24 & 23 & $\mathrm{n}+\mathrm{x}$ \\
\hline $\mathrm{n}$ & 25 & 26 & n-xy \\
\hline $\mathrm{n}$ & 26 & 25 & $\mathrm{n}+\mathrm{xy}$ \\
\hline
\end{tabular}




\section{Numerical investigation on $3 \mathrm{D}$ air explosion}

\subsection{Numerical investigation on air explosion for TNT charge}

In this subsection, simulations of air explosion for the TNT charge are performed utilizing a parallel high order code described previously. Explosion products are described by the JWL EOS, and its parameters can be found in Table 1. The initial pressure of the explosion products achieved in this subsection is $8.6 \mathrm{GPa}$. The computational model can be seen in Fig. 6 for the air explosion.

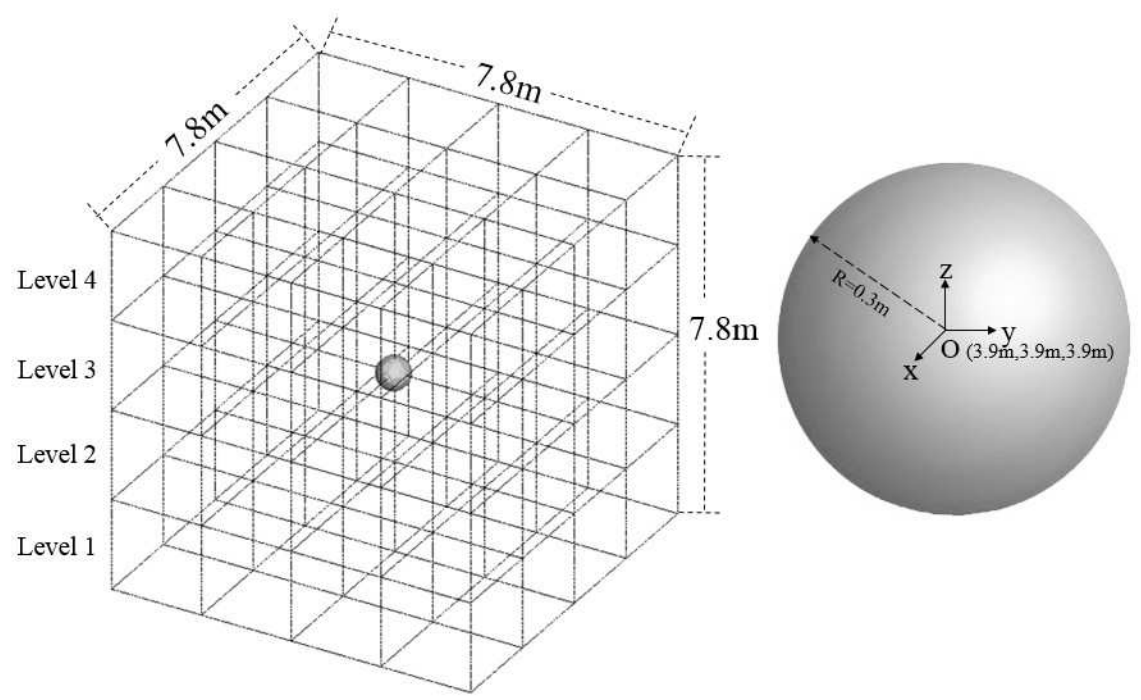

Fig. 6: Parallel computational model for the air explosion (left) and zoom-in on the explosive (right).

Before performing a series of numerical simulations, convergence of the computational code for the air explosion is validated. The computational domain with a TNT charge radius of $0.3 \mathrm{~m}$ is $7.8 \mathrm{~m} \times 7.8 \mathrm{~m} \times 7.8 \mathrm{~m}$. To accelerate the speed of computations, 64 processors are employed to compute this problem. Numerical convergence verifications for the pressure have been carried out by using three different mesh sizes of $0.01 \mathrm{~m}, 0.02 \mathrm{~m}$ and $0.03 \mathrm{~m}$. Not only the history of the pressure with time at the location $(5.6 \mathrm{~m}, 3.9 \mathrm{~m}, 3.9 \mathrm{~m})$ but also the variation of the pressure in space along the $x$ direction for the fixed time $t=0.91 \mathrm{~ms}$ are given in Fig. 7. It is observed clearly from Fig. 7 that numerical convergence can be obtained with a mesh size of $0.02 \mathrm{~m}$.

In order to demonstrate and validate the reliability of the numerical results, explosion wave overpressures obtained at different locations are compared with results derived from 

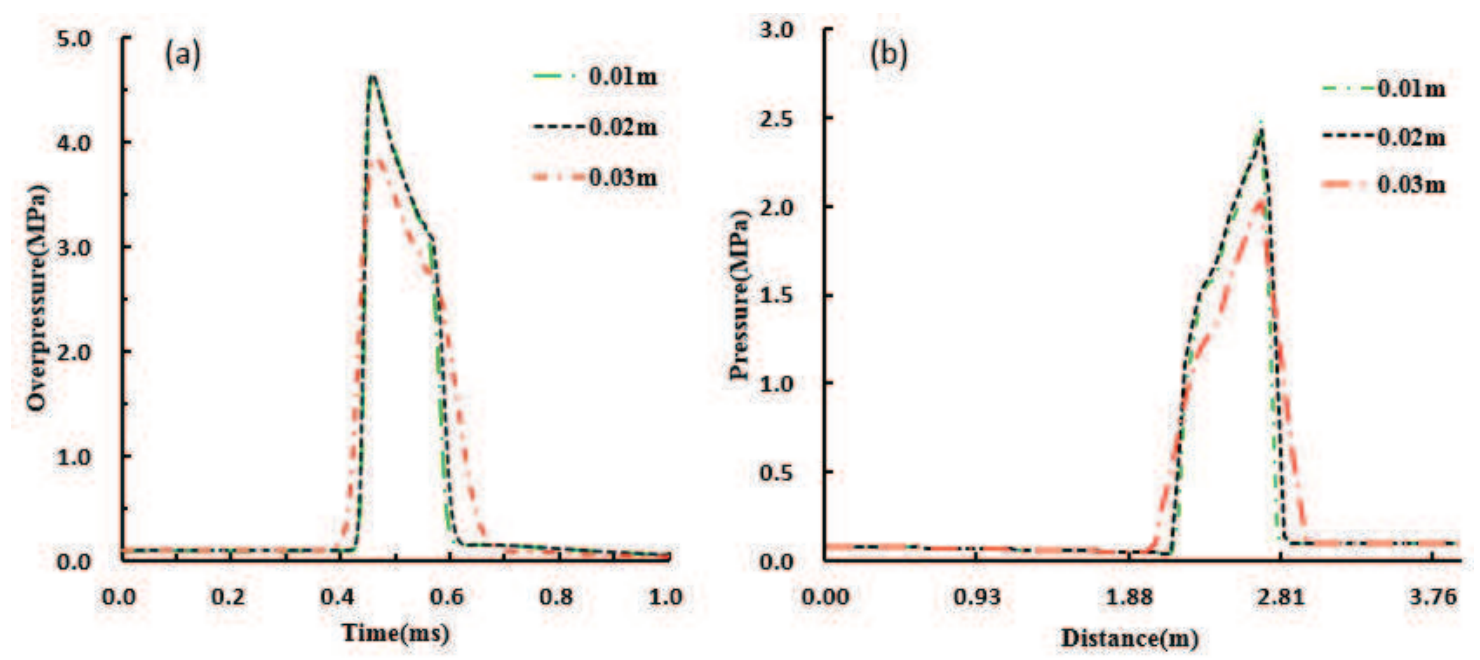

Fig. 7: Validations of convergence: (a) Pressure histories at the same location; (b) Pressure curves along the $x$ direction at the same instant.

the empirical formula presented by Henrych in [28] and simulated in the spherical coordinates, respectively. In the cartesian coordinates, 64 processors are employed to compute this problem with up to 59.78 million nodes. The size of a uniform cubic mesh is $0.02 \mathrm{~m} \times 0.02 \mathrm{~m} \times 0.02 \mathrm{~m}$, while the total physical domain with the TNT charge radius of $0.21 \mathrm{~m}$ is also $7.8 \mathrm{~m} \times 7.8 \mathrm{~m} \times 7.8 \mathrm{~m}$. In the spherical coordinate computation, we use an extremely refined mesh with the mesh size of $0.0001 \mathrm{~m}$, and keep other computational conditions the same as those in the cartesian coordinates. The comparison result is shown in Fig. 8. It is clear that, except for the region of the near field (in which the scaled distance is less than $\left.0.348 \mathrm{~m} / \mathrm{kg}^{1 / 3}\right)$, explosion wave overpressures obtained by the numerical simulation are in good agreement with the empirical formula, with a relative error less than 8.3 percent. It is also clear that the result computed in the cartesian coordinates is consistent with that computed in the spherical coordinates with an extremely refined mesh. As a result, the material interface treatment combining the level-set technique with the RGFM method based on a shock-shock Riemann solver can be applied to the numerical simulation of air explosion. At the same time, the comparison also shows that neglecting the details of the reaction process, by simplifying the explosive products into a high temperature and high pressure zone, seems to have little effect on the final result of the far field explosion overpressure.

From a mathematical viewpoint, the expansion process of the interface is the movement 


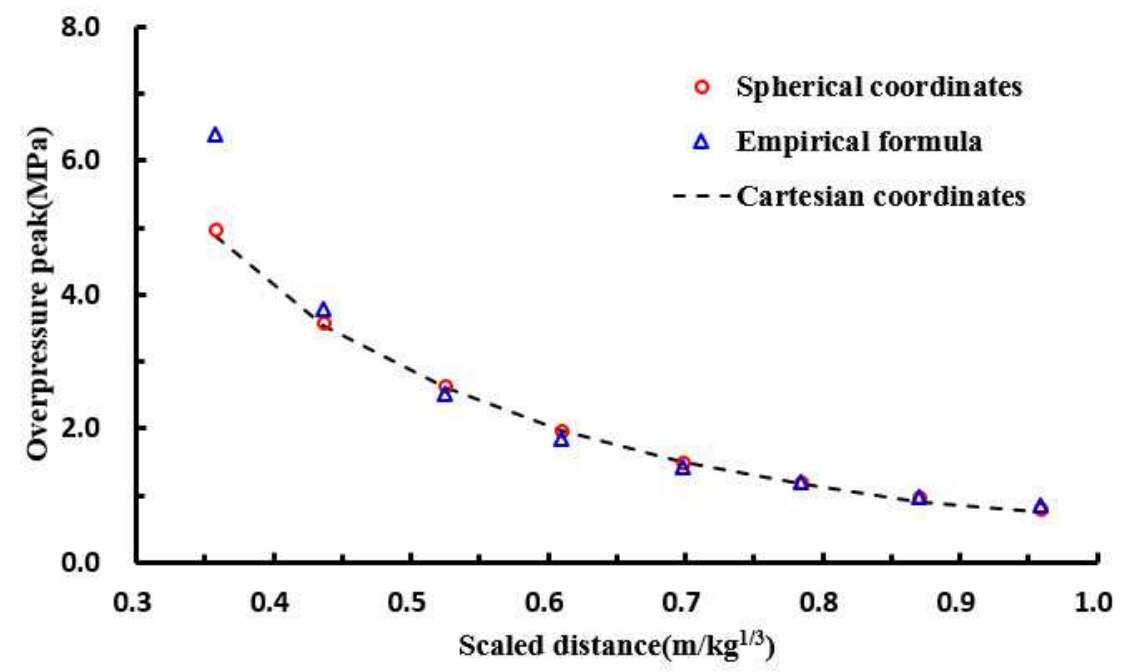

Fig. 8: Comparison of the explosion wave overpressure peaks by the cartesian coordinates, spherical coordinates (with an extremely refined mesh) and an empirical formula.

of a contact discontinuity decided by the interfacial velocity. Thus, solving a local Riemann problem is an important technique to simulate the evolution of air explosion. Results at several temporal moments are shown in Figs. 9 to 11 . In order to observe directly the distributions of the physical variables such as the pressure and density inside the 3D explosion field, results in levels 2 and 3 of the computational domain are shown. The distributions of density and pressure in the air explosion field are revealed clearly. It can also be seen from Fig. 12 that the evolution of pressure are symmetrical and nonphysical oscillation do not appear in the neighborhood of the interface.
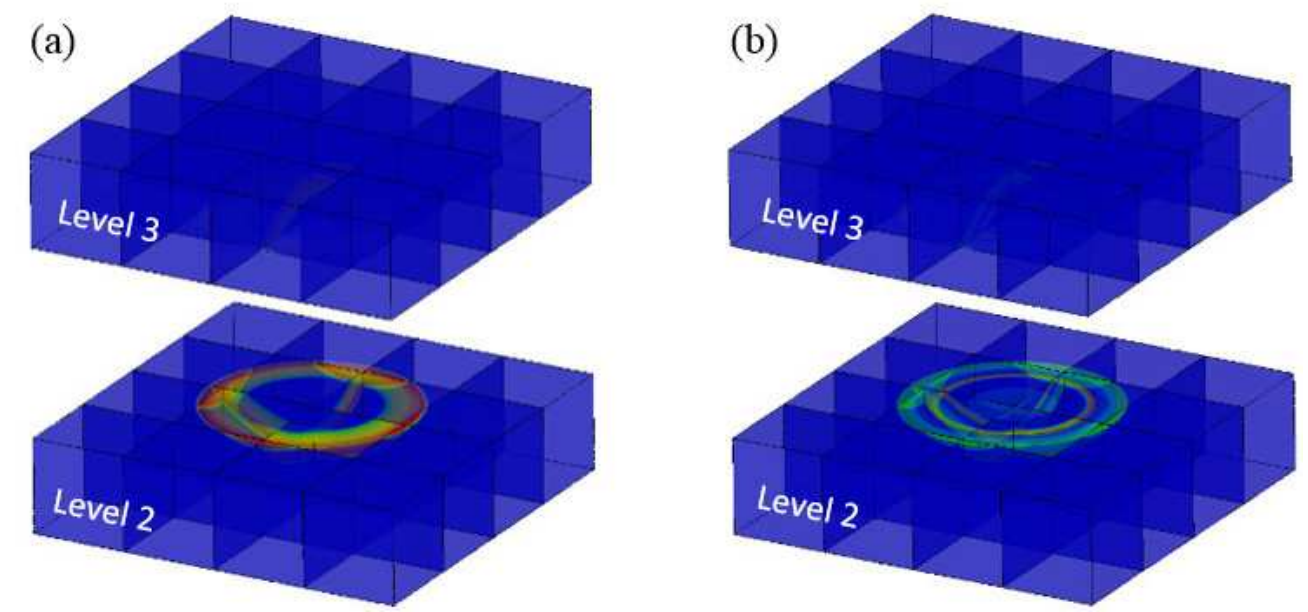

Fig. 9: Numerical results at 0.50ms: (a) Pressure contour; (b) Density contour. 

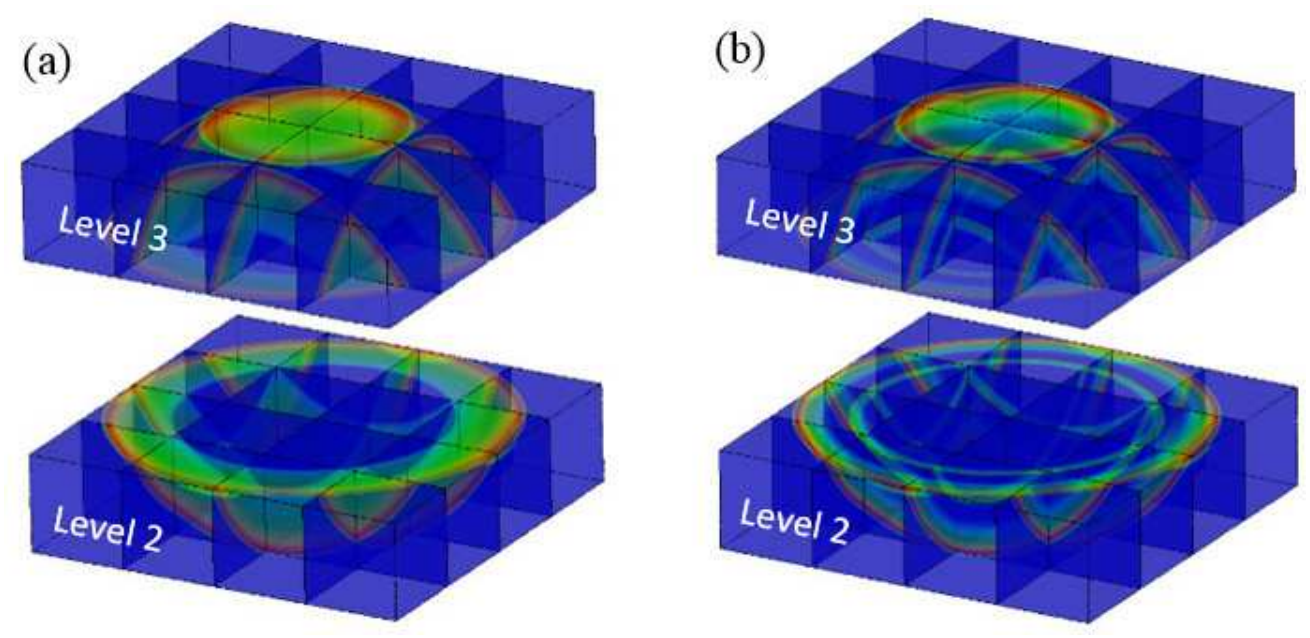

Fig. 10: Numerical results at 1.01ms: (a) Pressure contour; (b) Density contour.
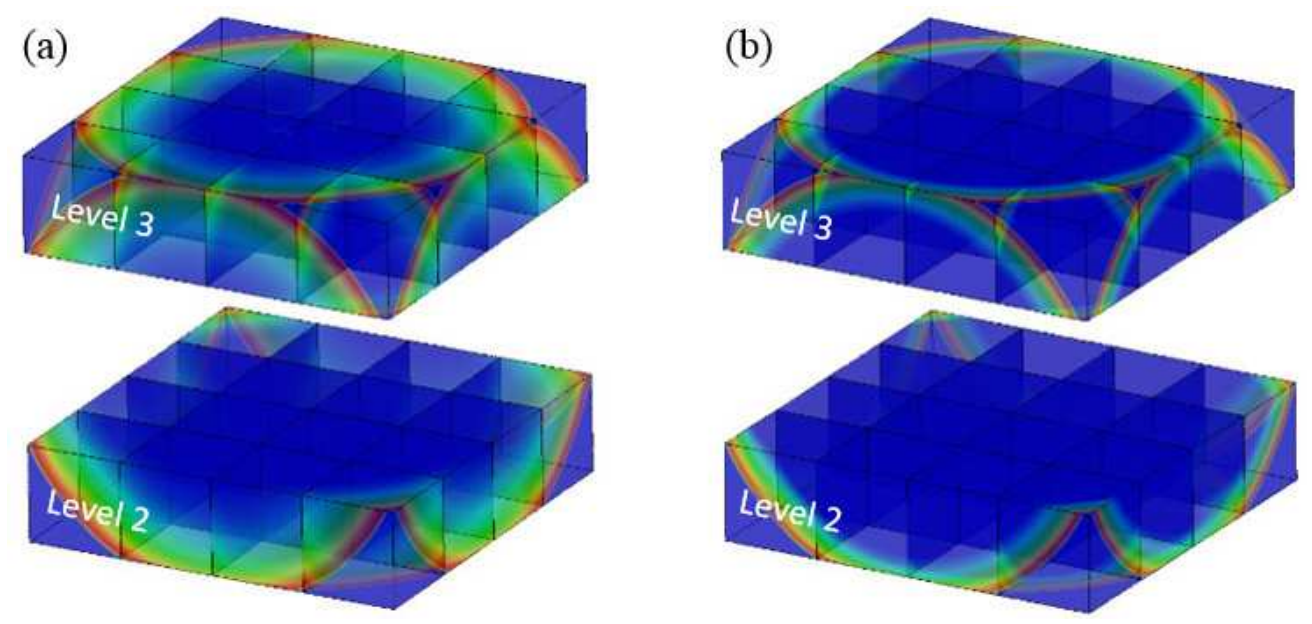

Fig. 11: Numerical results at 1.71ms: (a) Pressure contour; (b) Density contour. 


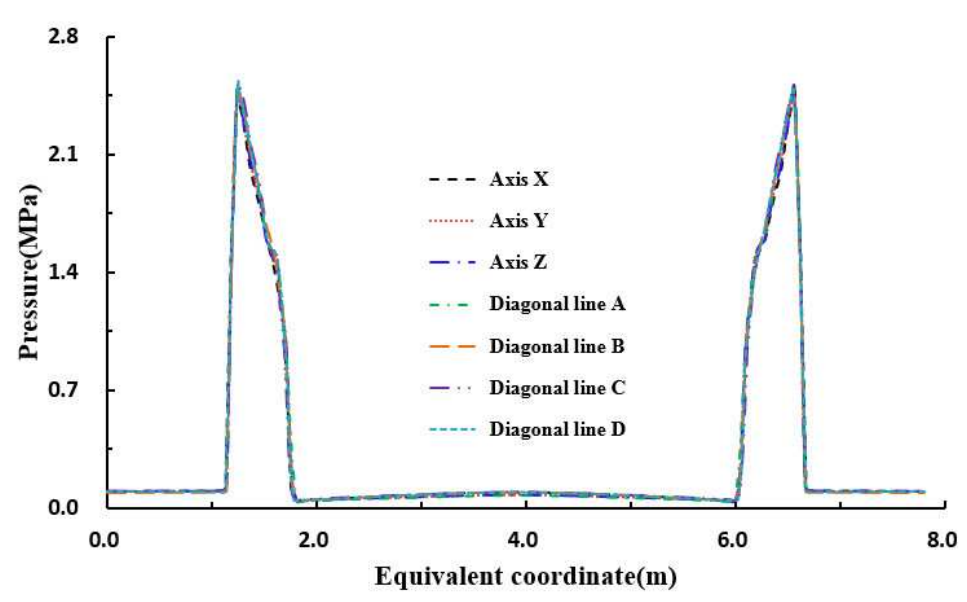

Fig. 12: Pressure distributions along the $x, y$ and $z$ directions and the 4 diagonal directions: Axis X denotes from the back to front; Axis Y denotes from the left to right; Axis Z denotes from the bottom to top; Diagonal line A denotes from the top back left to bottom front right; Diagonal line B denotes from the top back right to bottom front left; Diagonal line $\mathrm{C}$ denotes from the top front left to bottom back right, and Diagonal line D denotes from the top front right to bottom back left.

Fig. 13 shows the distributions of pressure and density for the spherical explosion field along the central axis at $0.772 \mathrm{~ms}$. It is obvious that there are two density peaks in Fig. 13(b). The first peak is inside the explosion products close to the interface, while the other appears in the explosion wave front in the air. The curve describing the evolution of pressure has only one peak, and pressure is continuous at the interface. It can also be found that, in the rapid expansion process of the air pushed by the explosion products with extremely high temperature and pressure, the rarefaction wave propagates inward and converges to the center of spherical explosion products, which dramatically attenuates pressure in the zones swept by the rarefaction wave. At $0.772 \mathrm{~ms}$, density distributes from $0.5 \mathrm{~kg} / \mathrm{m}^{3}$ to 1.0 $\mathrm{kg} / \mathrm{m}^{3}$ before the first peak, which is slightly lower than that under ambient temperature and pressure; pressure is less than 0.1 MPa, which is also below the normal atmospheric pressure. However, a narrow band with relatively high pressure and density is located inside the explosion products and borders the propagating interface. In this band, the maximum pressure and density can reach up to $0.86 \mathrm{MPa}$ and $3.3 \mathrm{~kg} / \mathrm{m}^{3}$, respectively, which are much higher than the atmospheric ones under ambient temperature and pressure. 

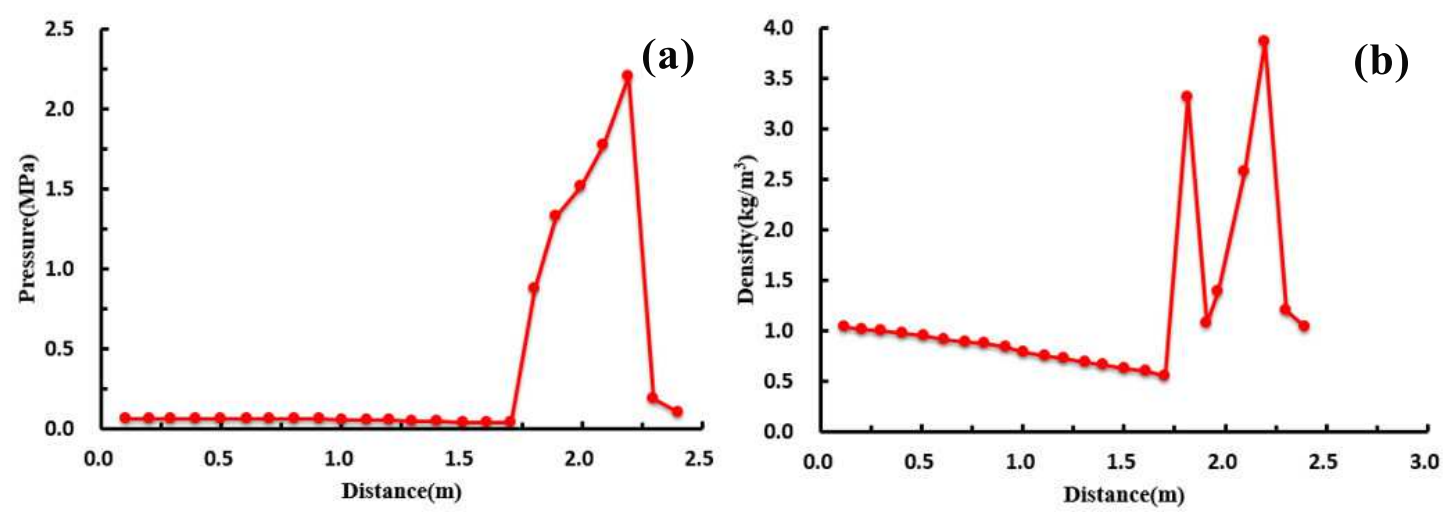

Fig. 13: Distributions of pressure and density along the central axis at 0.772ms: (a) Pressure curve; (b) Density curve.
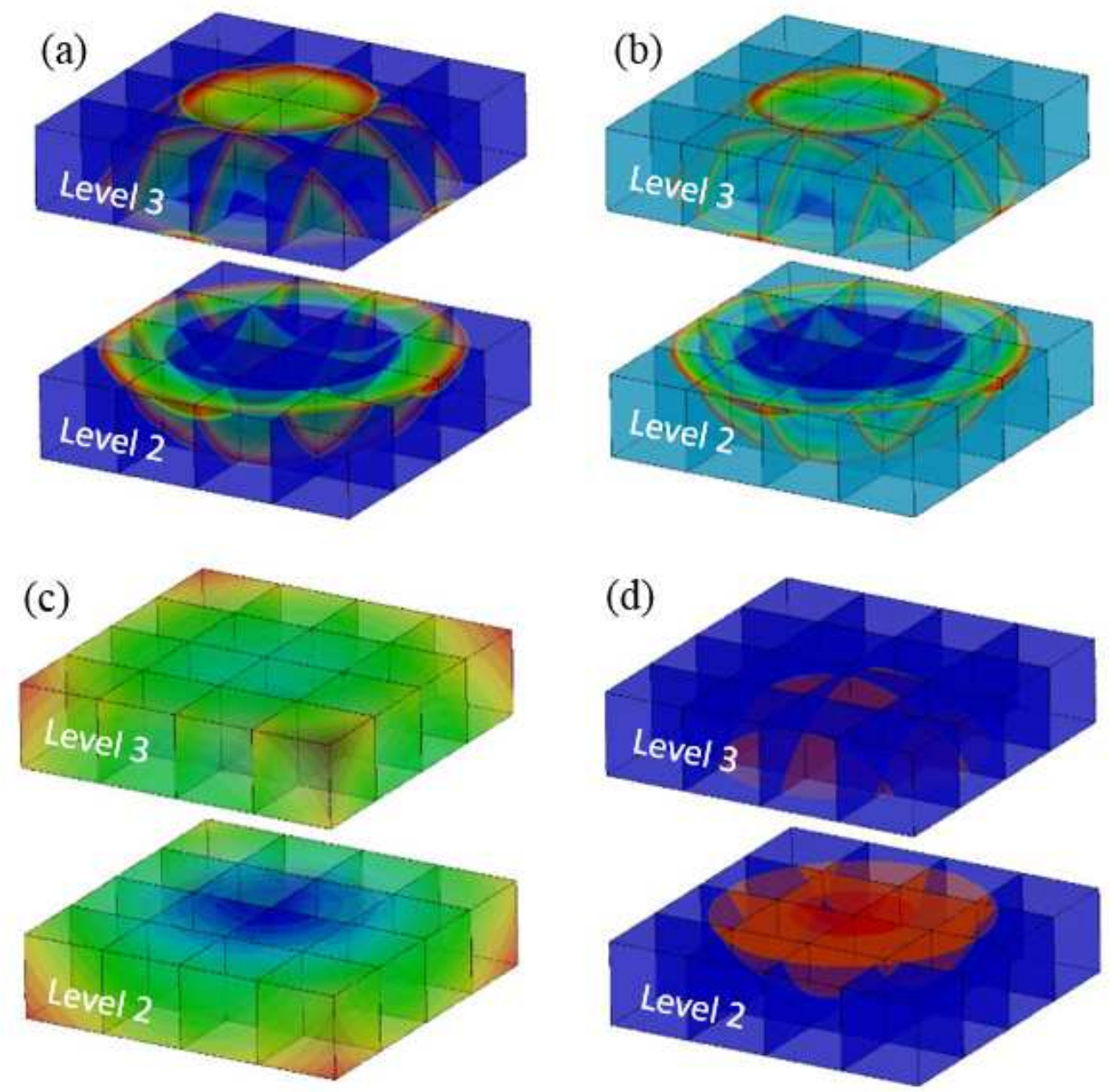

Fig. 14: Numerical results for the aluminized explosives: (a) Pressure contour; (b) Density contour; (c) Level-set contour; (d) Aluminum powder reaction fraction contour. 


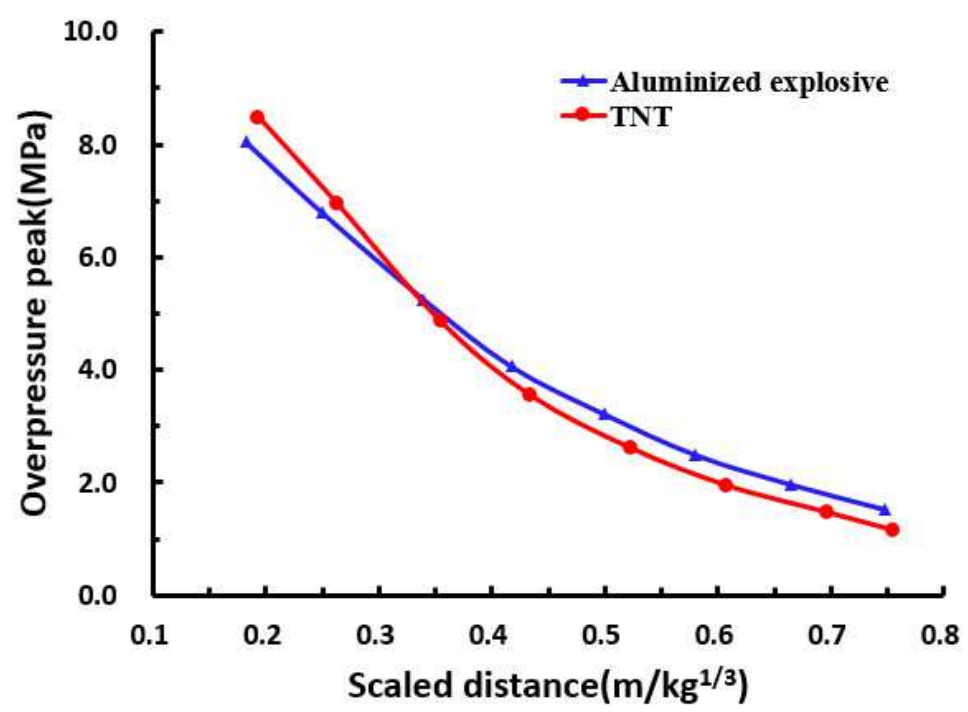

Fig. 15: Comparison of explosion wave overpressure peaks between TNT and aluminized explosives in air explosion.

\subsection{Numerical investigation on air explosion of aluminized explosives}

In this subsection, simulations of air explosion for aluminized explosives with the radius of $0.21 \mathrm{~m}$ are performed. The JWL-Miller EOS is used here to describe the explosion products, and Table 2 gives all parameters used. For simplicity, the ignition and reaction process of aluminized explosives are again omitted in the air explosion. The initial pressure of the explosion products is $6.26 \mathrm{GPa}$. Numerical results are again obtained with 64 processors and the total number of nodes is 59.78 million. The mesh size is $0.02 \mathrm{~m} \times 0.02 \mathrm{~m} \times 0.02 \mathrm{~m}$ and the computational domain is $7.8 \mathrm{~m} \times 7.8 \mathrm{~m} \times 7.8 \mathrm{~m}$.

Fig. 14 shows the contours for two parts of the computational domain for aluminized explosives. Table 4 shows the evolution of the explosion wave overpressure peak for the aluminized explosive with the change of the scaled distance. Fig. 15 compares the explosion wave overpressure peaks of TNT charge with that of aluminized explosives. It can be seen from Fig. 15 that the explosion wave overpressure peaks caused by aluminized explosive are obviously lower than that of TNT in the near field. However, because the secondary energy release caused by aluminum powder afterburning can continuously supplement energy, the energy loss in the propagating process of the explosion wave is replenished to some extent. Therefore, explosion wave attenuation of aluminized explosives is relatively slow, and its 
Tab. 4: The explosion wave overpressure peaks for aluminized explosive at different scaled distances.

\begin{tabular}{lccccccccc}
\hline $\begin{array}{l}\text { Scaled } \\
\text { distance }\left(\mathrm{m} / \mathrm{kg}^{1 / 3}\right)\end{array}$ & 0.182 & 0.249 & 0.332 & 0.415 & 0.497 & 0.580 & 0.663 & 0.746 & 0.829 \\
\hline $\begin{array}{l}\text { Overpressure } \\
\text { peak }(\mathrm{MPa})\end{array}$ & 8.09 & 6.85 & 5.31 & 4.10 & 3.22 & 2.52 & 1.97 & 1.55 & 1.25 \\
\hline
\end{tabular}

overpressure gradually catches up with and then surpasses that formed by TNT as the explosion wave spreads outward to the far field.

\section{Concluding remarks}

Combining the RGFM multi-medium interface treatment method with a fifth order finite difference WENO scheme, and based on a Riemann solver presented for nonlinear EOS and MPI-based enclosed-type parallel module, a large-scale parallel code is developed to simulate the 3D air explosion processes for TNT charge and aluminized explosives. First of all, air explosion of 3D spherical TNT charge is computed numerically, and then distributions of physical quantities such as density and pressure are obtained in the explosive flow field. Validation of the code based on the RGFM for simulating air explosion is given through comparing explosion wave overpressures obtained in the cartesian coordinates with Henrych empirical formula [28] and results from spherical coordinates. The errors are less than 8.3 percent except for the region in the near field close to the interface in air. At the same time, it is also demonstrated that, neglecting the reaction process in air explosion, as is done in this paper, seems to have little impact on the final numerical results of far field explosion overpressure. Next, air explosion of the spherical aluminized explosives is simulated to obtain the pressure and density distributions in the explosion field. The overpressure peaks of TNT charge and aluminized explosives are compared, which shows that the subsequent aluminum powder afterburning gives rise to the secondary energy release and forces relatively slow attenuation of explosion wave overpressure peaks of aluminized explosives. 


\section{Acknowledgments}

The research of Wang, Ding and Li is supported by the National Natural Science Foundation of China under grants 11325209, 11272056 and 11521062. The research of Shu is supported by ARO grant W911NF-15-1-0226 and NSF grant DMS-1418750.

\section{References}

[1] F.H. Harlow and J.E. Welch. Numerical calculation of time dependent viscous incompressible flow of fluid with free surface. Phys. Fluids, 1965, 8:2182-2189.

[2] C.W. Hirt and B.D. Nichols. Volume of fluid (VOF) method for the dynamics of free boundaries. J. Comput. Phys., 1981, 39:201-225.

[3] J. Zhang, J. Fang and B.Q. Fan. Advances in research of VOF method. Adv. Sci. Technol. Water Resour., 2005,25:67-70.

[4] H. Ahn and M. Shashkov. Multi-material interface reconstruction on generalized polyhedral meshes. J. Comput. Phys., 2007, 226:2096-2132.

[5] V. Dyadechko and M. Shashkov. Reconstruction of multi-material interfaces from moment data. J. Comput. Phys., 2008, 227:5361-5384.

[6] A. Amsden. The particle-in cell method for the calculations of the dynamics of compressible fluids. Los Alamos Scientific Laboratory Report, 1966, LA-3466.

[7] S. Osher and J.A. Sethian. Fronts propagating with curvature dependent speed: algorithms based on Hamilton-Jacobi formulations. J. Comput. Phys., 1988, 79:12-49.

[8] M. Vahab and G. Miller. A front-tracking shock-capturing method for two gases. Comm. App. Math. Com. Sc., 2015, 11:1-35.

[9] S. Osher and R. Fedkiw. Level set methods and dynamic implicit surfaces. Springer, New York, 2003.

[10] J.A. Sethian. Theory, algorithms, and applications of level set methods for propagating interfaces. Acta Numer., 1996, 5:309-395. 
[11] D. Adalsteinsson and J.A. Sethian. The fast construction of extension velocities in level set methods. J. Comput. Phys., 1999, 148:2-22.

[12] R. Fedkiw, T. Aslam, B. Merriman and S. Osher. A non-oscillatory Eulerian approach to interfaces in multimaterial flows (the ghost fluid method). J. Comput. Phys., 1999, 152:457-492.

[13] T.G. Liu, B.C. Khoo and K.S. Yeo. Ghost fluid method for strong shock impacting on material interface. J. Comput. Phys., 2003, 190:651-681.

[14] T.G. Liu and B.C. Khoo. The accuracy of the modified ghost fluid method for gas-gas Riemann problem. Appl. Numer. Math., 2007, 57:721-733.

[15] C.W. Wang, T.G. Liu and B.C. Khoo. A real ghost fluid method for the simulation of multimedium compressible flow. SIAM. J. Sci. Comput., 2006, 28:278-302.

[16] P.J. Miller. A reactive flow model with coupled reaction kinetics for detonation and combustion in non-ideal explosives. Mat. Res. Soc. Symp. Proc., 1996, 418:413-420.

[17] E.L. Lee, H.C. Hornig and J.W. Kury. Adiabatic expansion of high explosive detonation products. UCRL Report, 1968, UCRL-50422.

[18] G.S. Jiang and C.-W. Shu. Efficient implementation of weighted ENO schemes. J. Comput. Phys., 1996, 126:202-228.

[19] C.-W. Shu and S. Osher. Efficient implementation of essentially non-oscillatory shockcapturing schemes. J. Comput. Phys., 1988, 77:439-471.

[20] C. Wang, X.Z. Dong and C.-W. Shu. Parallel adaptive mesh refinement method based on WENO finite difference scheme for the simulation of multi-dimensional detonation. J. Comput. Phys., 2015, 298:161-175.

[21] G.-S. Jiang and D. Peng. Weighted ENO schemes for Hamilton-Jacobi equations. SIAM J. Sci. Comput., 2000, 21:2126-2143. 
[22] R. Nourgaliev and T. Theofanous. High-fidelity interface tracking in compressible flows: unlimited anchored adaptive level set. J. Comput. Phys., 2007, 224:836-866.

[23] M. Sussman, P. Smereka and S. Osher. A level set approach for computing solutions to incompressible two-phase flow. J. Comput. Phys., 1994, 114:146-159.

[24] P.A. Gremaud, C.M. Kuster and Z.L. Li. A study of numerical methods for the level set approach. Appl. Numer. Math., 2007, 57:837-846.

[25] D. Hartmann, M. Meinke and W. Schröder. Differential equation based constrained reinitialization for level set methods. J. Comput. Phys., 2008, 227:6821-6845.

[26] D. Hartmann, M. Meinke and W. Schröder. The constrained reinitialization equation for level set methods. J. Comput. Phys., 2010, 229:1514-1535.

[27] T.D. Aslam. A partial differential equation approach to multidimensional extrapolation. J. Comput. Phys., 2003, 193:349-355.

[28] J. Henrych. The dynamics of explosion and its use. Elsevier Scientific Publishing Company, Amsterdam and New York, 1979, 178-181. 